\title{
SEPARATION OF ELECTRONIC AND NUCLEAR CONTRIBUTIONS TO THE THIRD-ORDER NONLINEAR OPTICAL SUSCEPTIBILITIES OF NEW TETRATHIAFULVALENE DERIVATIVES USING DEGENERATE FOUR WAVE MIXING
}

\author{
B. Sahraoui*, X. NguYen Phu
}

Laboratoire des Propriétés Optiques des Matériaux et Applications: POMA CNRS EP 130, Université d'Angers, 2, Boulevard Lavoisier, 49045 Angers cedex, France

\section{SALLÉ AND A. GorguES}

Laboratoire d'Ingénierie Moléculaire Matériaux et Organiques, UMR CNRS 6501 Université d'Angers, 2, Boulevard Lavoisier, 49045 Angers cedex, France

We separate the electronic and nuclear contributions to the third-order nonlinear optical susceptibilities $\chi_{i j k l}^{\langle 3\rangle}$ using degenerate four wave mixing. This method allows to know the physical origin of the optical nonlinearities of new soluble tetrathiafulvalene derivatives and deduce the values of the second-order hyperpolarisabilities $\gamma$. The values of $\gamma$ for the studied compounds are about $10^{5}$ greater than for $\mathrm{CS}_{2}$.

PACS numbers: $42.65 . \mathrm{Ky}, 78.66 . \mathrm{Qn}, 42.70 . \mathrm{Nq}$

Organic molecules characterised by large $\pi$-electron systems over an extended and conjugated framework are of interest for their strong nonlinear optical properties [1-3]. The molecular structure of various series of tetrathiafulvalene (TTF) derivatives makes them suitable candidates to reveal large third-order nonlinear optical susceptibilities [3]. In this paper we present a relatively simple method, based on degenerate four wave mixing (DFWM) measurements, to distinguish different physical mechanisms contributing to the third-order susceptibilities. We study and compare some ethylenic and $p-N, N^{\prime}$-dimethylaniline TTF derivatives. The chemical structure of the studied molecules are presented in Fig. 1 and their UV spectra in Fig. 2. The measurements of transmission for the range of intensities used $\left(0-1 \mathrm{GW} / \mathrm{cm}^{2}\right)$ show that the molecules present only linear absorption.

\footnotetext{
*e-mail: sahraoui@univ-angers.fr
} 
<smiles>CC1=C(C)SC(=CC=CC=C2SC(C)=C(C)S2)S1</smiles>

(b)<smiles>CN(C)c1ccc(/C=C/C2=C(/C=C/c3ccc(N(C)C)cc3)SC(=C3SC(/C=C/c4ccc(N(C)C)cc4)=C(/C=C/c4ccc(N(C)C)cc4)S3)S2)cc1</smiles><smiles>C/C=C\C=C\C1=C(/C=C/c2ccc(N(C)C)cc2)SC(=C2SC3=C(SCCS3)S2)S1</smiles>

Fig. 1. Chemical structures of (a) ethylenic of TTF derivative, (b) and (c) $p-N, N^{\prime}$-dimethylaniline of TTF derivatives.

The DFWM experimental arrangement is illustrated in Fig. 3. The excitation is provided by $30 \mathrm{ps}$ laser pulses at $\lambda=532 \mathrm{~nm}$ generated by an amplified mode locked Quantel Nd:YAG laser operating at $1 \mathrm{~Hz}$ repetition. Two of the interacting waves are strong counterpropagating pumps travelling in the forward and backward directions. Their intensities verify the relation $I_{1}(z=0)=I_{2}(z=l)$. The third input beam is a weak probe $\left(I_{3}, I_{4} \ll I_{1}\right)$ which makes an angle $12^{\circ}$ with respect to the pump. Phase matching requires then that the signal wave be radiated in a direction backward to the probe. The thickness of the cell containing the solution of the powdered organic molecules is $0.1 \mathrm{~cm}$.

Using Maxwell nonlinear equations one can obtain the DFWM efficiency in the form [4]:

$$
R_{i j k l}=\frac{I^{\langle 4\rangle}(0)}{I^{\langle 3\rangle}(0)}= \begin{cases}\frac{p^{2}+\frac{\alpha^{2}}{4}}{\left[p \operatorname{ctg}(p l)+\frac{\alpha}{2}\right]^{2}}, & p^{2} \geq 0, \\ \frac{p^{2}+\frac{\alpha^{2}}{4}}{\left[q \operatorname{cth}(q l)+\frac{\alpha}{2}\right]^{2}}, & p^{2}<0,\end{cases}
$$

where $p^{2}=\left(\frac{48 \pi^{3}}{n^{2} c \lambda} \chi_{i j k l}^{\langle 3\rangle}\right)^{2} I^{\langle 1\rangle}(0)^{2} \exp (-\alpha l)-\frac{\alpha^{2}}{4}, q=\mathrm{i} p$ and $l$ is the cell length. The DFWM efficiency $R$ with the subscripts $i j k l$ indicates that the polarisation states of the waves $\langle 4\rangle,\langle 1\rangle,\langle 2\rangle$ and $\langle 3\rangle$ are $i, j, k, l$ respectively which take values $x$ or $y \cdot \chi_{i j k l}^{(3)}$ values can be obtained by adjusting the theoretical curve given by (1) to the measured values of $R$.

We measured $I^{(4)}$ versus the concentration of molecules in the solution in order to determine the optimal concentration $C_{\text {opt }}$ at which $I^{(4\rangle}$ is maximum. All the compounds investigated displayed a single maximum in $I^{(4)}$. An example 


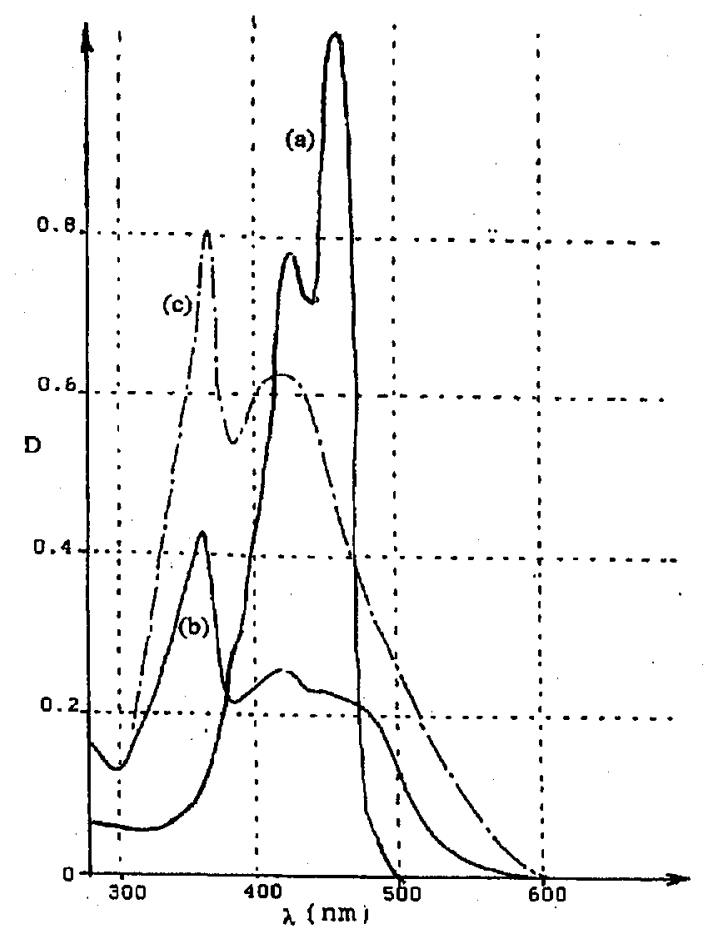

Fig. 2. UV spectra of studied compounds: (a) ethylenic of TTF derivative, (b) and (c) $p$ - $N, N^{\prime}$-dimethylaniline of TTF derivatives. These spectra measurements were performed with a cell of $1 \mathrm{~cm}$ thick at very weak concentrations $\left(\approx 10^{-5} \mathrm{~mol} / \mathrm{dm}^{3}\right)$.

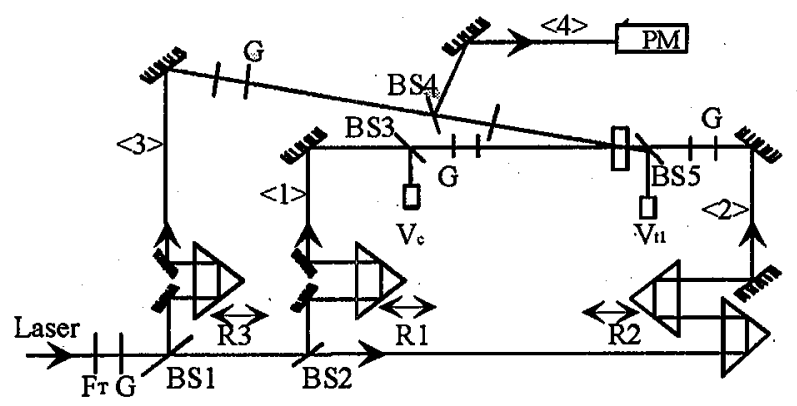

Fig. 3. Experimental setup. $\langle 1\rangle$ and $\langle 2\rangle-$ pumps beams, $\langle 3\rangle$ - probe beam, $\langle 4\rangle-$ fourth beam, $S$ - sample, $F_{\mathrm{T}}-$ neutral density filter, $G-$ Glan prism, $V_{\mathrm{t}}-$ control photodiodes, $V_{\mathrm{c}}-c$ photodiode, $R_{1,2,3}$ - delay lines, $B S_{1,2,3,4}$ - beam splitters and $P M$ - photomultiplier tube.

of these results (for compound (b)) is presented in Fig. 4. $C_{\text {opt }}$ values and the absorption coefficients at $C_{\text {opt }}$ are collected in Table I.

We measured the DFWM efficiency $R$ at $C_{\text {opt }}$ as a function of wave $\langle 1\rangle$ intensity for various polarization states of waves $\langle 1\rangle,\langle 2\rangle$ and $\langle 3\rangle$. Figure 5 shows 

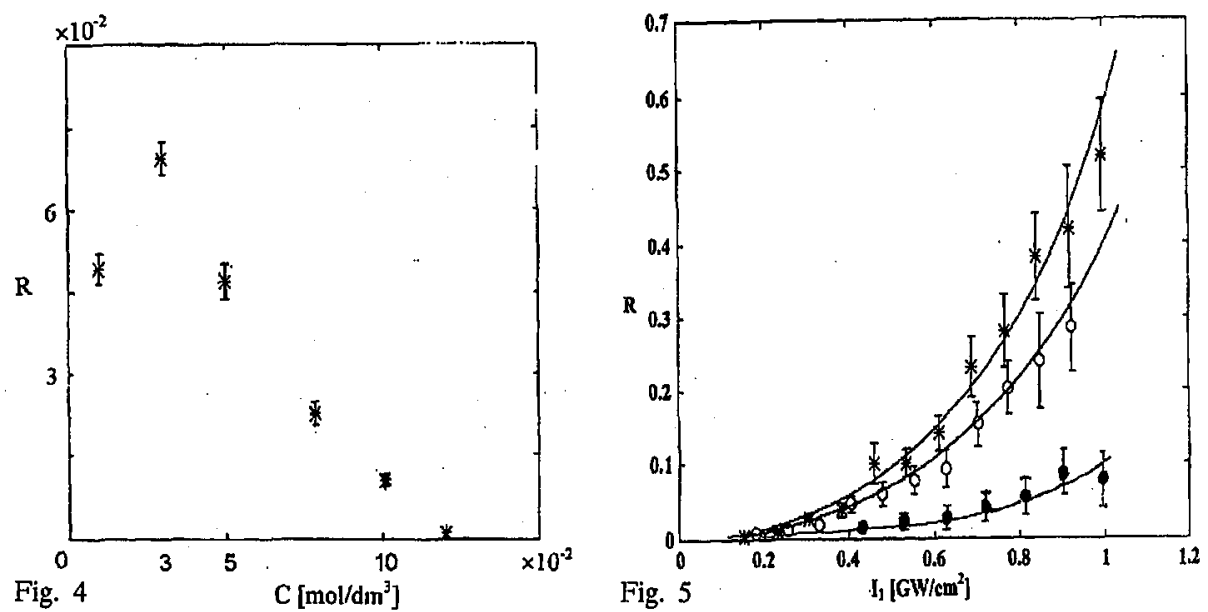

Fig. 4. Dependence of degenerate four waves mixing efficiency on solution concentration $C$. A typical curve is shown for the ethylenic TTF derivative (a) dissolved in chloroform. The polarization state of incident wave $\langle 1\rangle,\langle 2\rangle$ and $\langle 3\rangle$ is vertical $(x x x)$ and their intensities satisfy the relations: $I^{\langle 1\rangle}(z=0)=I^{\langle 2\rangle}(z=l)$ and $I^{\langle 3\rangle}(z=0)=10^{-2} I^{\langle 1\rangle}(z=0)$.

Fig. 5. Degenerate four wave mixing efficiency versus intensity of the pumps $\langle 1\rangle$ for the compounds (a) (•), (b) (*) and (c) (o). The polarization state of incident waves $\langle 1\rangle,\langle 2\rangle$ and $\langle 3\rangle$ is vertical $(x x x)$ and the continuous curve corresponds to the theoretical formula (1).

\section{TABLE I}

Parameters $M, C_{\text {opt }}, \alpha$ represent respectively molar weight, the optimum concentration and linear absorption coefficient at $C_{\text {opt }}$ respectively.

\begin{tabular}{c|c|c|c}
\hline \hline Molecules & $\begin{array}{c}M \\
{[\mathrm{~g} / \mathrm{mol}]}\end{array}$ & $\begin{array}{c}C_{\text {opt }} \\
{\left[\mathrm{mol} / \mathrm{dm}^{3}\right]}\end{array}$ & $\begin{array}{c}\alpha \\
{\left[\mathrm{cm}^{-1}\right]}\end{array}$ \\
\hline (a) & 312 & $3 \times 10^{-2}$ & 21.4 \\
(b) & 584 & $1.3 \times 10^{-3}$ & 16.8 \\
(c) & 784 & $0.7 \times 10^{-3}$ & 20.6
\end{tabular}

the results for the three compounds studied in the case of vertical polarization of the incident beams. The adjustment of the theoretical curve given by (1) to experimental data leads to the third-order susceptibility for the compounds (Table II). From the results, we can conclude that the following relationships are verified:

for molecule (a): $\quad \chi_{x x x x}^{\exp } \approx 2 \chi_{x y x y}^{\exp } \approx 2 \chi_{x x y y}^{\exp } \approx 10 \chi_{x y y x}^{\exp }$,

for molecule (b): $\quad \chi_{x x x x}^{\exp } \approx 2.3 \chi_{x y x y}^{\exp } \approx 2.3 \chi_{x x y y}^{\exp } \approx 8.8 \chi_{x y y x}^{\exp }$,

for molecule (c): $\quad \chi_{x x x x}^{\exp } \approx 2.7 \chi_{x y x y}^{\exp } \approx 2.7 \chi_{x x y y}^{\exp } \approx 4.1 \chi_{x y y x}^{\exp }$. 
TABLE II

$\chi^{\langle 3\rangle}$ components deduced from degenerate four wave mixing experiment for the new TTF derivatives (a) and (b) studied at their optimum concentration.

\begin{tabular}{|c|c|c|c|c|c|}
\hline Molecules & $\begin{array}{c}\left|\chi_{x x x x}^{\exp }\right| \\
\times 10^{20} \\
{\left[\mathrm{~V}^{2} \mathrm{~m}^{-2}\right]}\end{array}$ & $\begin{array}{c}\left|\chi_{x y x y}^{\exp }\right| \\
\times 10^{20} \\
{\left[\mathrm{~V}^{2} \mathrm{~m}^{-2}\right]}\end{array}$ & $\begin{array}{c}\left|\chi_{x x y y}^{\exp }\right| \\
\times 10^{20} \\
{\left[\mathrm{~V}^{2} \mathrm{~m}^{-2}\right]}\end{array}$ & $\begin{array}{c}\left|\chi_{x y y x}^{\exp }\right| \\
\times 10^{20} \\
{\left[\mathrm{~V}^{2} \mathrm{~m}^{-2}\right]}\end{array}$ & $\begin{array}{c}\gamma_{x x x x} \\
\times 10^{45} \\
{\left[\mathrm{~m}^{5} \mathrm{~V}^{-2}\right]}\end{array}$ \\
\hline (a) & 8.0 & 4.1 & 4.0 & 0.8 & 1.1 \\
\hline (b) & 9.5 & 4.1 & 4 & 1.1 & 18 \\
\hline (c) & 11.4 & 4.2 & 4.1 & 2.8 & 71 \\
\hline
\end{tabular}

In the DFWM experiences, we have used a laser delivering $30 \mathrm{ps}$ pulses so the thermal and electrostrictive effects could be neglected, because they are very slow. In this case two essential local effects contribute to the nonlinearities in isotropic materials excited by such laser pulses: electronic cloud deformation and nucleus reorientation (translations, rotations, and vibrations). As a consequence, one can consider $\chi^{\langle 3\rangle}$ as consisting of two contributions corresponding to the mentioned mechanisms: $\chi_{i j k l}^{\langle 3\rangle}=\chi_{i j k l}^{\langle 3\rangle \mathrm{el}}+\chi_{i j k l}^{\langle 3 / \mathrm{nu}}$. One can show that for isotropic media the electronic and nuclear tensorial components satisfy the relations [5]:

$$
\begin{aligned}
& \chi_{x x x x}^{\langle 3\rangle \mathrm{el}}=3 \chi_{x x y y}^{\langle 3\rangle \mathrm{el}}=3 \chi_{y x y x}^{\langle 3\rangle \mathrm{el}}=3 \chi_{y x x y}^{\langle 3\rangle \mathrm{el}}, \\
& \chi_{x x x x}^{\langle 3\rangle \mathrm{nu}}=8 \chi_{x x y y}^{\langle 3\rangle \mathrm{nu}}=8 \chi_{y x y x}^{\langle 3\rangle \mathrm{nu}}=\frac{4}{3} \chi_{y x x y}^{\langle 3\rangle \mathrm{nu}} .
\end{aligned}
$$

Using the experimental results described by $(2 \mathrm{a}, 2 \mathrm{~b}, 2 \mathrm{c})$ and the relations (3), one can deduce the following:

$$
\begin{array}{ll}
\text { for molecule }(\mathrm{a}): \quad \chi_{x x x x}^{\mathrm{el}} \approx 164 \chi_{x x x x}^{\exp }, & \chi_{x x x x}^{\mathrm{nu}} \approx-0.64 \chi_{x x x x}^{\exp }, \\
\text { for molecule }(\mathrm{b}): \quad \chi_{x x x x}^{\mathrm{el}} \approx 1.53 \chi_{x x x x}^{\exp }, & \chi_{x x x x}^{\mathrm{nu}} \approx-0.53 \chi_{x x x x}^{\exp }, \\
\text { for molecule }(\mathrm{c}): \quad \chi_{x x x x}^{\mathrm{el}} \approx 1.2 \chi_{x x x x}^{\exp }, & \chi_{x x x x}^{\mathrm{nu}} \approx-0.2 \chi_{x x x x}^{\exp } .
\end{array}
$$

Hence, we can conclude that the electronic contribution is larger than the nuclear contribution for the molecules of interest and it is negative. For molecule (c) the electronic contribution is larger than for molecules (a) and (b) which probably due to the fact that molecule (c) has more an extended and conjugated structure which favours a high $\pi$-electron delocalisation. Moreover, the (c) molecule possesses the biggest molar weight, which causes smaller rotation, vibration effects and in turn gives a relatively small nuclear contribution. To establish the sign of the nonlinearities, we performed additional experimental test which has showed that the studied solutions are defocussing. This fact allows us to conclude that the $\chi^{\langle 3\rangle}$ is negative. Hence, the electronic component of $\chi^{\langle 3\rangle}$ is negative.

Second-order hyperpolarisability characterizing an individual molecule is related to the third-order susceptibility by [2]:

$$
\chi^{\langle 3\rangle}=F^{4} N \gamma_{\text {solution }}+\chi_{\text {solvent }}^{\langle 3\rangle},
$$


where $F=\left(n^{2}+2\right) / 3$ is the Lorentz field factor correction, $N=N_{\mathrm{A}} \cdot C / M$ is the number of solution molecules per unity of volume; $N_{\mathrm{A}}$ is the Avogadro number. In our case, $\chi_{\text {solvent }}^{\langle 3\rangle}$ can be neglected because it is very small compared with $\chi_{\text {solution }}^{\langle 3\rangle}$ at the concentrations used. The second-order hyperpolarisabilities are presented in Table II.

By DFWM and by changing the polarisation states of the interacting beams, we are able to determine the origin of the third-order optical nonlinearities by separation of electronic and nuclear contributions to $\chi_{\text {solution }}^{(3)}$. For all the molecules studied, we found that the electronic component of $\chi^{(3)}$ is dominant and its sign is negative. For molecule (c) the electronic third-order susceptibility is larger than for molecules (a) and (b). This can be due to a more extended and fully conjugated structure which favours a $\pi$-electron delocalisation. The molecules investigated possess large third-order nonlinearities compared, for example, to polyazine derivatives [6]. The second-order hyperpolarisabilities are about $10^{5}$ times greater than in $\mathrm{CS}_{2}$ [7], which is a reference material for DFWM measurements.

\section{References}

[1] F. Kajzar, J.D. Swalem, Organic Thin Films for Waveguiding Nonlinear Optic, Vol. 3, Gordon and Breach, 1996.

[2] J. Zyss, D.S. Chemla, Nonlinear Optical Properties of Organic Molecules and Crystals, Vol. 1, 2, Quantum Electronics (Principles and Applications), Academic, Orlando 1987.

[3] B. Sahraoui, M. Sylla, J.P. Bourdin, G. Rivoire, J. Zaremba, T.T. Nguyen, M. Sallé, J. Mod. Opt. 42, 2095 (1995).

[4] B. Sahraoui, G. Rivoire, Opt. Commun. 138, 109 (1997).

[5] J.P. Bourdin, P.X. Nguyen, G. Rivoire, J.M. Nunzi, Nonlinear Opt. 7, 1 (1994).

[6] H.S. Nalwa, T. Hamada, A. Kakuta, A. Mukoh, Synth. Metals 55, 3901 (1993).

[7] R.W. Boyd, Nonlinear Optics, Academic, New York 1992. 